\title{
Gravitational radiation reaction in compact binary systems: Contribution of the quadrupole-monopole interaction
}

\author{
László Á. Gergely and Zoltán Keresztes \\ Astronomical Observatory and Department of Experimental Physics, \\ University of Szeged, Dóm tér 9, Szeged, H-6720 Hungary
}

\begin{abstract}
The radiation reaction in compact spinning binaries on eccentric orbits due to the quadrupolemonopole interaction is studied. This contribution is of second post-Newtonian order. As result of the precession of spins the magnitude $L$ of the orbital angular momentum is not conserved. Therefore a proper characterization of the perturbed radial motion is provided by the energy $E$ and angular average $\bar{L}$. As powerful computing tools, the generalized true and eccentric anomaly parametrizations are introduced. Then the secular losses in energy and magnitude of orbital angular momentum together with the secular evolution of the relative orientations of the orbital angular momentum and spins are found for eccentric orbits by use of the residue theorem. The circular orbit limit of the energy loss agrees with Poisson's earlier result.
\end{abstract}

\section{INTRODUCTION}

Compact binary systems composed of neutron stars and/or black holes are among the best candidates of sources emitting gravitational radiation in the frequency range of the Earth-based interferometric detectors such as the Laser Interferometric Gravitational Wave Observatory (LIGO) [1], VIRGO [2], GEO [3], and TAMA [4], and also for the envisaged Laser Interferometer Space Antenna (LISA) [5.6. Before the final coalescence of the compact binary due to a cumulative loss in energy and angular momentum there is a regime where a post-Newtonian description of the motion and of the gravitational radiation is well suited, provided it is taken with sufficient accuracy.

As up to the second post-Newtonian (PN) order the energy and total angular momentum of the system are conserved, and the leading contribution of gravitational radiation at 2.5 PN is generally agreed to decrease the eccentricity of orbits faster than their radii [7, it is customary to compute radiation templates and the radiation reaction with the assumption of circular orbits. Another argument for such a simplifying assumption is the reduction of the number of fitting parameters in the matched filtering. However as described in Refs. [8] and [9], there are various astrophysical situations in galactic nuclei where the eccentricity of the orbits is still significant. The error one makes in searching for waves emitted by binaries on circular orbits when the waves come from binaries on eccentric orbits was estimated to be substantial in [10]. Therefore it is desirable to have a generic treatment, also valid for eccentric orbits. Such a treatment, valid up to the second post-Newtonian order was provided by Gopakumar and Iyer [11].

But the situation is even more complicated. At 1.5 PN order other features of the binary appear. These are spinorbit type contributions, which further increase the number of parameters to be fitted. At $2 \mathrm{PN}$ both spin-spin and quadrupole-monopole effects appear. The latter are the consequences of considering each component of the binary as a monopole in the quadrupolar field of the other. The nonradiative dynamics of spinning binaries with emphasis on both the spins and the quadrupole-monopole interaction was investigated long ago in a series of papers by Barker and O'Connell (see, for example, [12]). Radiative aspects concerning spins were studied in Refs. [13]- [19]. However the quadrupole-monopole type radiative contribution has received less attention until now, excepting the circular orbit limit, discussed in detail by Poisson [20]. In this paper we extend this treatment to eccentric orbits.

For this purpose in Sect. II we derive two parametrizations of the orbit, which generalize the Keplerian true and eccentric anomaly parametrizations for the case of the quadrupole-monopole perturbation. These parametrizations have the advantageous property that the required integral expressions can be easily computed by use of the residue theorem and, further, in the majority of cases the only pole is at the origin [21]. Our treatment follows closely and relies on the results of Refs. [18] and [19]. Here we also find the peculiar feature encountered previously solely in the discussion of the spin-spin contributions, that the magnitude of the orbital angular momentum is not a conserved quantity. We bypass this difficulty by introducing its angular average $\bar{L}$ and characterizing the perturbed orbit by $E$ and $\bar{L}$. It is equally possible to characterize the motion by $E$ and the time average $\langle L\rangle$; however the resulting expressions would be more cumbersome. For completeness, the relation between $\langle L\rangle$ and $\bar{L}$ is given. At the end of the section we find the same functional expression for the period of the radial motion as in the unperturbed case, but with the energy characterizing the perturbed motion. This is a consequence of the specific functional form of the perturbing term in the radial equation [21].

Having developed the required toolchest, the computation of the quadrupole-monopole contributions to the losses of energy and magnitude of orbital angular momentum together with the evolution of the angle variables, which characterize the relative orientation of the spin vectors and orbital angular momentum vector are straightforward. 
These are given in Sect. III as the main results of the paper. We will show in the concluding remarks that in the circular limit the energy loss reduces to the expression given previously by Poisson.

The velocity of light $c$ and the gravitational constant $G$ are kept in all expressions.

\section{THE RADIAL MOTION}

The Keplerian motion of the binary perturbed by the monopole-quadrupole interaction between its components is properly characterized by the Lagrangian [20], 22]:

$$
\begin{aligned}
\mathcal{L} & =\mathcal{L}_{N}+\mathcal{L}_{Q M} \\
\mathcal{L}_{N} & =\frac{\mu \mathbf{v}^{2}}{2}+\frac{G m \mu}{r} \\
\mathcal{L}_{Q M} & =\frac{G \mu m^{3}}{2 r^{5}} \sum_{i=1}^{2} p_{i}\left[3\left(\hat{\mathbf{S}}_{\mathbf{i}} \cdot \mathbf{r}\right)^{2}-r^{2}\right]
\end{aligned}
$$

where $\mathcal{L}_{N}$ represents the Newtonian part of the Lagrangian (with $m$ the total mass and $\mu$ the reduced mass). The summation is taken over the two bodies in the quadrupole-monopole interaction term $\mathcal{L}_{Q M}$, which depends both on the direction $\hat{\mathbf{S}}_{\mathbf{i}}$ of the spin of the $i^{\text {th }}$ body $\mathbf{S}_{\mathbf{i}}$ and on $p_{i}$, defined as

$$
p_{i}=\frac{Q_{i}}{m_{i} m^{2}} .
$$

Here $Q_{i}$ is the quadrupole-moment scalar [20] of the $i^{\text {th }}$ axially symmetric binary component with symmetry axis $\hat{\mathbf{S}}_{\mathbf{i}}$. In the Newtonian limit computation gives

$$
Q_{i}=\Theta_{i}^{\prime}-\Theta_{i}=-\frac{S_{i}}{\Omega_{i}}\left(\frac{\Theta_{i}}{\Theta_{i}^{\prime}}-1\right)
$$

for each axisymmetric body characterized by the principal moments of inertia $\left(\Theta_{i}, \Theta_{i}, \Theta_{i}^{\prime}\right)$ and angular velocity $\Omega_{i}=S_{i} / \Theta_{i}^{\prime}$.

Other contributions, like PN, 2PN [11], spin-orbit (SO) 17] and spin-spin (SS) [18], [19] appearing at 1.5 and second post-Newtonian orders, respectively will add to the dynamics. However these other post-Newtonian terms will not interfere with the quadrupole-monopole interaction terms. Quadrupole-monopole terms are singled out from among the rest of the second post-Newtonian order terms by the distinguishing parameters $p_{i}$ in the same way spin-spin interaction terms can be recognized as being quadratic in the spin magnitudes. Therefore up to the second postNewtonian order each contribution can be computed independently, including the quadrupole-monopole one. In fact the first interference terms appear in the 2.5 order, as the first PN correction of the SO-terms [23].

The acceleration resulting from Eq. (2.1) is

$$
\begin{aligned}
\mathbf{a}= & \mathbf{a}_{N}+\mathbf{a}_{Q M} \\
\mathbf{a}_{N}= & -\frac{G m \mathbf{r}}{r^{3}} \\
\mathbf{a}_{Q M}= & -\frac{3 G m^{3}}{2 r^{7}} \sum_{i=1}^{2} p_{i}\left[5\left(\hat{\mathbf{S}}_{\mathbf{i}} \cdot \mathbf{r}\right)^{2}-r^{2}\right] \mathbf{r} \\
& +\frac{3 G m^{3}}{r^{5}} \sum_{i=1}^{2} p_{i}\left(\hat{\mathbf{S}}_{\mathbf{i}} \cdot \mathbf{r}\right) \hat{\mathbf{S}}_{\mathbf{i}}
\end{aligned}
$$

By performing the required changes in notations the acceleration $\mathbf{a}_{Q M}$ coincides with Eqs. (55)-(56) of Ref. [12].

The Lagrangian governing the quadrupole-monopole interaction is not time dependent; therefore the total energy $E$ of the system is conserved. We further remark that, as it happens for spin-spin interaction, here the Lagrangian $\mathcal{L}_{Q M}$ has no dependence on velocities either. Therefore $\mathbf{p}=\mu \dot{\mathbf{r}}$ and two conclusions emerge:

(a.) The energy is given by

$$
E=E_{N}-\mathcal{L}_{Q M}
$$


(b.) There is no quadrupole-monopole contribution to the orbital angular momentum

$$
\mathbf{L}=\mathbf{r} \times \mathbf{p}=\mathbf{L}_{N} .
$$

The functional form of the Newtonian energy and orbital angular momentum vector 18] allows one to relate them to $v^{2}$ and $\dot{r}^{2}$ :

$$
\begin{aligned}
& v^{2}=\frac{2 E_{N}}{\mu}+\frac{2 G m}{r}, \\
& \dot{r}^{2}=\frac{2 E_{N}}{\mu}+\frac{2 G m}{r}-\frac{L_{N}^{2}}{\mu^{2} r^{2}} .
\end{aligned}
$$

The cross product of Eq. (2.4 with $\mu$ r gives the evolution of the orbital angular momentum:

$$
\dot{\mathbf{L}}=\frac{3 G \mu m^{3}}{r^{5}} \sum_{i=1}^{2} p_{i}\left(\hat{\mathbf{S}}_{\mathbf{i}} \cdot \mathbf{r}\right)\left(\mathbf{r} \times \hat{\mathbf{S}}_{\mathbf{i}}\right)
$$

Due to the quadrupole-monopole interaction the spins undergo a precessional motion, computed a long time ago by Barker and O'Connell [Eqs. (39) and (43) of [12] referring to bodies with arbitrary, but constant mass, spin and quadrupole moment]:

$$
\dot{\mathbf{S}}_{\mathbf{i}}=-\frac{3 G \mu m^{3}}{r^{5}} p_{i}\left(\hat{\mathbf{S}}_{\mathbf{i}} \cdot \mathbf{r}\right)\left(\mathbf{r} \times \hat{\mathbf{S}}_{\mathbf{i}}\right) .
$$

It is then straightforward to check the conservation of the total angular momentum

$$
\mathbf{J}=\mathbf{L}+\mathbf{S}_{\mathbf{1}}+\mathbf{S}_{\mathbf{2}} .
$$

Multiplying Eq. (2.9) by the direction of the orbital angular momentum $\hat{\mathbf{L}}$ the magnitude of the angular momentum is found to evolve. The nonconservation of the magnitude of the orbital angular momentum is a feature already encountered when studying spin-spin dynamics. By performing the transformations enlisted in Ref [18] the detailed form of the evolution of $L$ emerges

$$
\dot{L}=-\frac{3 G \mu m^{3}}{2 r^{3}} \sum_{i=1}^{2} p_{i} \sin ^{2} \kappa_{i} \sin 2\left(\psi-\psi_{i}\right) .
$$

Here and hereafter we employ the following angles. $\kappa_{i}=\cos ^{-1}\left(\hat{\mathbf{S}}_{\mathbf{i}} \cdot \hat{\mathbf{L}}\right)$ and $\gamma=\cos ^{-1}\left(\hat{\mathbf{S}}_{\mathbf{1}} \cdot \hat{\mathbf{S}}_{\mathbf{2}}\right)$ characterize the relative orientation of the angular momenta. The angles $\psi$ and $\psi_{i}$ are subtended by the intersection line of the planes perpendicular to $\mathbf{J}$ and $\mathbf{L}$ with the position $\mathbf{r}$ and the projections of the spins in the plane of the orbit, respectively [see Fig. 1 in Ref. [17]. We also introduce $\delta_{i}=2\left(\psi_{0}-\psi_{i}\right)$ ]. This description allows for computing the value of $L$ in terms of the Newtonian true anomaly parameter $\chi=\psi-\psi_{0}$ and a properly defined angular average $\bar{L}$ of $L$ in a similar way as was done in the spin-spin case:

$$
\begin{aligned}
L(\chi)= & \bar{L}+\delta L \\
\delta L= & \frac{G \mu^{3} m^{3}}{4 \bar{L}^{3}} \sum_{i=1}^{2} p_{i} \sin ^{2} \kappa_{i}\left\{2 \bar{A} \cos \left(\chi+\delta_{i}\right)\right. \\
& \left.+(3 G \mu m+2 \bar{A} \cos \chi) \cos \left(2 \chi+\delta_{i}\right)\right\} .
\end{aligned}
$$

where

$$
\bar{A}=\left(G^{2} m^{2} \mu^{2}+\frac{2 E \bar{L}^{2}}{\mu}\right)^{1 / 2}
$$

is the magnitude of the Laplace-Runge-Lenz vector for a Keplerian motion characterized by $E$ and $\bar{L}$.

By following the method of Ref. [18] the quadrupole-monopole interaction part $E_{Q M}$ of the energy can also be given in terms of $\chi$ and $r$ : 


$$
E_{Q M}(r, \chi)=\frac{G \mu m^{3}}{2 r^{3}} \sum_{i=1}^{2} p_{i}\left[1-3 \sin ^{2} \kappa_{i} \cos ^{2}\left(\chi+\frac{\delta_{i}}{2}\right)\right],
$$

Then the expressions (2.7) and (2.8) take the form:

$$
\begin{aligned}
& v^{2}=\frac{2\left[E-E_{Q M}(r, \chi)\right]}{\mu}+\frac{2 G m}{r}, \\
& \dot{r}^{2}=\frac{2\left[E-E_{Q M}(r, \chi)\right]}{\mu}+\frac{2 G m}{r}-\frac{L(\chi)^{2}}{\mu^{2} r^{2}},
\end{aligned}
$$

with $L(\chi)$ and $E_{Q M}(r, \chi)$ given by Eqs. (2.13) and (2.15). As the parameter $\chi$ appears only in second post-Newtonian terms, it can be replaced by any generalized true anomaly parameter having the proper Newtonian limit. In what follows we will seek for such a suitable parametrization $r=r(\chi)$ of the radial component of the perturbed motion.

For this purpose we compute the turning points of the orbit, defined as roots of the radial equation (2.17) with $\dot{r}^{2}=0$ for $\chi=0, \pi$. We find:

$$
\begin{aligned}
r_{\max } & =\frac{G m \mu \pm \bar{A}}{-2 E}+\frac{G \mu^{2} m^{3}}{4 \bar{A} \bar{L}^{2}} \sum_{i=1}^{2} p_{i} \rho_{\mp}^{i}, \\
\rho_{\mp}^{i} & =\alpha_{0}^{i}(\bar{A} \mp G m \mu)+\beta_{0}^{i}(4 \bar{A} \mp 3 G m \mu), \\
\alpha_{0}^{i} & =2\left[1-3 \sin ^{2} \kappa_{i} \cos ^{2}\left(\psi_{0}-\psi_{i}\right)\right], \\
\beta_{0}^{i} & =\sin ^{2} \kappa_{i} \cos 2\left(\psi_{0}-\psi_{i}\right) .
\end{aligned}
$$

Then, following Ref. 18 we derive the generalized true anomaly parametrization

$$
r=\frac{\bar{L}^{2}}{\mu(G m \mu+\bar{A} \cos \chi)}+\frac{G \mu^{2} m^{3}}{4 \bar{A} \bar{L}^{2}(G m \mu+\bar{A} \cos \chi)^{2}} \sum_{i=1}^{2} p_{i} \Lambda^{i},
$$

with

$$
\begin{aligned}
\Lambda^{i} & =\bar{A}\left[\bar{A}^{2}\left(\alpha_{0}^{i}+4 \beta_{0}^{i}\right)+(G m \mu)^{2}\left(3 \alpha_{0}^{i}+10 \beta_{0}^{i}\right)\right] \\
& +G m \mu\left[\bar{A}^{2}\left(3 \alpha_{0}^{i}+11 \beta_{0}^{i}\right)+(G m \mu)^{2}\left(\alpha_{0}^{i}+3 \beta_{0}^{i}\right)\right] \cos \chi
\end{aligned}
$$

In a similar way we derive the generalized eccentric anomaly parametrization

$$
r=\frac{G m \mu-\bar{A} \cos \xi}{-2 E}+\frac{G \mu^{2} m^{3}}{4 \bar{A} \bar{L}^{2}} \sum_{i=1}^{2} p_{i} \Xi^{i},
$$

with

$$
\Xi^{i}=\bar{A}\left(\alpha_{0}^{i}+4 \beta_{0}^{i}\right)+G m \mu\left(\alpha_{0}^{i}+3 \beta_{0}^{i}\right) \cos \xi .
$$

Note that $\rho_{\mp}^{i}, \Lambda^{i}$, and $\Xi^{i}$ have the same structure as in the spin-spin case; however, the angular expressions $\alpha_{0}^{i}$ and $\beta_{0}^{i}$ are different from $\alpha_{0}$ and $\beta_{0}$ introduced in Ref. [18].

In the perturbative terms we will need the Keplerian relations

$$
\dot{r}=\frac{\bar{A}}{\bar{L}} \sin \chi, \quad \dot{\psi}=\frac{\bar{L}}{\mu r^{2}} .
$$

The two parametrizations are suitable for averaging radial expressions by use of the residue theorem, as described in detail in [21]. The time average of $L(\chi)$ is

$$
\langle L\rangle=\bar{L}+\frac{G m^{3} \mu^{2} F_{1}}{4 \bar{A}^{2} \bar{L}^{3} F_{2}} \sum_{i=1}^{2} p_{i} \sin ^{2} \kappa_{i} \cos \delta_{i}
$$

with the coefficients $F_{1,2}$ given by 


$$
\begin{aligned}
F_{1}= & 2 \bar{L}(-2 \mu E)^{1 / 2}\left[\bar{A}^{6}-15 G^{2} m^{2} \mu^{2} \bar{A}^{4}+32 G^{4} m^{4} \mu^{4} \bar{A}^{2}-16 G^{6} m^{6} \mu^{6}\right] \\
& +G m \mu^{2}\left[-11 \bar{A}^{6}+58 G^{2} m^{2} \mu^{2} \bar{A}^{4}-80 G^{4} m^{4} \mu^{4} \bar{A}^{2}+32 G^{6} m^{6} \mu^{6}\right] \\
F_{2}= & 4 G m \bar{L}(-2 \mu E)^{1 / 2}\left[\bar{A}^{2}-2 G^{2} m^{2} \mu^{2}\right]+\bar{A}^{4}-8 G^{2} m^{2} \mu^{2} \bar{A}^{2}+8 G^{4} m^{4} \mu^{4} .
\end{aligned}
$$

We remark that the coefficients $F_{1,2}$ are the same as the corresponding coefficients in the spin-spin case. As $L(\chi)$ given in terms of $\langle L\rangle$ has a more cumbersome expression than Eq. 2.13), we will give all forthcoming expressions in terms of $\bar{L}$.

For the period once again we find a Keplerian expression, but with $E$ characterizing the perturbed dynamics:

$$
T=2 \pi G m\left(\frac{\mu}{-2 E}\right)^{3 / 2}
$$

\section{LEADING ORDER QUADRUPOLE-MONOPOLE CONTRIBUTION TO THE EVOLUTIONS OF THE DYNAMICAL VARIABLES UNDER RADIATION REACTION}

\section{A. Energy loss}

To leading order the instantaneous loss of energy under radiation reaction is given by Einstein's quadrupole formula

$$
\frac{d E}{d t}=-\frac{G}{5 c^{5}} I^{(3) j l} I^{(3) j l}
$$

where $\epsilon^{i j k}$ is the completely antisymmetric Levi-Civita symbol, the numbers in parantheses denote a corresponding order time derivative and the system's symmetric trace-free (STF) mass quadrupole moment $I_{N}^{j l}$ to leading order is given by

$$
I_{N}^{j l}=\mu\left(x^{j} x^{l}\right)^{S T F} .
$$

The Newtonian and quadrupole-monopole contribution to the instantaneous loss under radiation reaction in the energy is :

$$
\frac{d E}{d t}=-\frac{G}{5 c^{5}} I_{N}^{(3) j l}\left(\mathbf{a}_{N}\right) I_{N}^{(3) k l}\left(\mathbf{a}_{N}\right)-\frac{2 G}{5 c^{5}} I_{N}^{(3) j l}\left(\mathbf{a}_{Q M}\right) I_{N}^{(3) k l}\left(\mathbf{a}_{N}\right) .
$$

The arguments of time derivatives of the momenta contain the contribution to the acceleration to be inserted in the respective terms. By inserting in the Newtonian terms the expressions (2.16) and (2.17) for $v^{2}$ and $\dot{r}^{2}$, respectively, and $L(\chi)$ and $E_{Q M}(r, \chi)$ given by Eqs. (2.13) and (2.15) and the Newtonian expressions in the quadrupole-monopople terms, we obtain:

$$
\begin{aligned}
\frac{d E}{d t} & =\left(\frac{d E}{d t}\right)_{N}+\left(\frac{d E}{d t}\right)_{Q M}, \\
\left(\frac{d E}{d t}\right)_{N} & =-\frac{8 G^{3} m^{2}}{15 c^{5} r^{6}}\left(2 \mu E r^{2}+2 G m \mu^{2} r+11 \bar{L}^{2}\right), \\
\left(\frac{d E}{d t}\right)_{Q M} & =\frac{2 G^{3} m^{4}}{15 c^{5} \bar{L}^{2} r^{8}} \sum_{i=1}^{2} p_{i}\left\{\sum_{n=1}^{3} a_{n} \cos \left(n \chi+\delta_{i}\right) \sin ^{2} \kappa_{i}+a_{4}\left(2-3 \sin ^{2} \kappa_{i}\right)\right\} .
\end{aligned}
$$

The coefficients $a_{k}$ are given by

$$
\begin{aligned}
& a_{1}=3 \mu \bar{A} r\left(-22 G m \mu^{2} r+17 \bar{L}^{2}\right), \\
& a_{2}=6\left(-11 G^{2} m^{2} \mu^{4} r^{2}+6 E \bar{L}^{2} \mu r^{2}+5 G m \mu^{2} \bar{L}^{2} r-51 \bar{L}^{4}\right), \\
& a_{3}=-\mu \bar{A} r\left(22 G m \mu^{2} r+51 \bar{L}^{2}\right), \\
& a_{4}=2 \bar{L}^{2}\left(-6 E \mu r^{2}-5 G m \mu^{2} r+39 \bar{L}^{2}\right) .
\end{aligned}
$$

By use of the true anomaly parametrization $r(\chi)$, Eq. (2.19) we find the energy loss in terms of $\chi$ alone. Then we pass to the complex parameter $z=\exp (i \chi)$ and we compute the averaged energy loss by use of the residue theorem, the only pole being at the origin: 


$$
\begin{aligned}
\left\langle\frac{d E}{d t}\right\rangle & =\left\langle\frac{d E}{d t}\right\rangle_{N}+\left\langle\frac{d E}{d t}\right\rangle_{Q M}, \\
\left\langle\frac{d E}{d t}\right\rangle_{N} & =-\frac{G^{2} m(-2 E \mu)^{3 / 2}}{15 c^{5} \bar{L}^{7}}\left(148 E^{2} \bar{L}^{4}+732 G^{2} m^{2} \mu^{3} E \bar{L}^{2}+425 G^{4} m^{4} \mu^{6}\right), \\
\left\langle\frac{d E}{d t}\right\rangle_{Q M} & =\frac{G^{2} \mu m^{3}(-2 E \mu)^{3 / 2}}{30 c^{5} \bar{L}^{11}} \sum_{i=1}^{2} p_{i}\left[C_{1} \sin ^{2} \kappa_{i} \cos \delta_{i}+C_{2}\left(2-3 \sin ^{2} \kappa_{i}\right)\right],
\end{aligned}
$$

with the coefficients $C_{k}$ given by:

$$
\begin{aligned}
& C_{1}=-\mu \bar{A}^{2}\left(948 E^{2} \bar{L}^{4}+8936 G^{2} m^{2} \mu^{3} E \bar{L}^{2}+8335 G^{4} m^{4} \mu^{6}\right) \\
& C_{2}=708 E^{3} \bar{L}^{6}+10020 G^{2} m^{2} \mu^{3} E^{2} \bar{L}^{4}+18865 G^{4} m^{4} \mu^{6} E \bar{L}^{2}+8316 G^{6} m^{6} \mu^{9} .
\end{aligned}
$$

\section{B. Change in the magnitude of orbital angular momentum}

The instantaneous and averaged losses in $L$ under the radiation reaction can be found in the same way as for the spin-spin case [18]:

$$
\frac{d L}{d t}=\hat{\mathbf{L}} \cdot \frac{d \mathbf{J}}{d t}-\hat{\mathbf{L}} \cdot \frac{d \mathbf{S}_{\mathbf{1}}}{d t}-\hat{\mathbf{L}} \cdot \frac{d \mathbf{S}_{\mathbf{2}}}{d t} .
$$

The evolution under radiation reaction in the spin $\mathbf{S}_{\mathbf{i}}$ of the $i^{t h}$ axisymmetric body (or approximately axisymmetric, with the deviation from axisymmetry of any post-Newtonian order), following [13] was derived in [24] by computing the integral of the moment of the reaction force (the sign swapped gradient of the Burke-Thorne potential) over the

volume of each body. The radiation reaction will change the instantaneous orientation but not the magnitude of the spin vectors. However in [19] it was shown that there is no secular spin evolution under radiation reaction in the $2 P N$ order:

$$
\left\langle\frac{d \mathbf{S}_{\mathbf{i}}}{d t}\right\rangle=0
$$

Denoting by $\simeq$ equalities modulo Burke-Thorne type terms, e.g. disregarding all terms which average out due to Eq.(3.13) we find for the instantaneous loss in $L$ under the radiation reaction:

$$
\frac{d L}{d t} \simeq \hat{\mathbf{L}} \cdot \frac{d \mathbf{J}}{d t} .
$$

The leading order instantaneous loss of $\mathbf{J}$ under radiation reaction is:

$$
\frac{d \mathbf{J}^{i}}{d t}=-\frac{2 G}{5 c^{5}} \epsilon^{i j k} I^{(2) j l} I^{(3) k l} .
$$

The Newtonian and quadrupole-monopole terms decouple as:

$$
\frac{d \mathbf{J}^{i}}{d t}=-\frac{2 G}{5 c^{5}} \epsilon^{i j k} I_{N}^{(2) j l}\left(\mathbf{a}_{N}\right) I_{N}^{(3) k l}\left(\mathbf{a}_{N}\right)-\frac{2 G}{5 c^{5}} \epsilon^{i j k}\left[I_{N}^{(2) j l}\left(\mathbf{a}_{N}\right) I_{N}^{(3) k l}\left(\mathbf{a}_{Q M}\right)+I_{N}^{(2) j l}\left(\mathbf{a}_{Q M}\right) I_{N}^{(3) k l}\left(\mathbf{a}_{N}\right)\right] .
$$

In the QM-terms we can use Cartesian coordinates $(x, y, z)=r(\cos \psi, \sin \psi, 0)$ [equivalent to $\hat{\mathbf{L}}=(0,0,1)]$. Then the spins are expressed as $\mathbf{S}_{\mathbf{i}}=S_{i}\left(\sin \kappa_{i} \cos \psi_{i}, \sin \kappa_{i} \sin \psi_{i}, \cos \kappa_{i}\right)$. The computation yields the following instantaneous losses:

$$
\begin{aligned}
\hat{\mathbf{L}} \cdot \frac{d \mathbf{J}}{d t} & =\left(\hat{\mathbf{L}} \cdot \frac{d \mathbf{J}}{d t}\right)_{N}+\left(\hat{\mathbf{L}} \cdot \frac{d \mathbf{J}}{d t}\right)_{Q M}, \\
\left(\hat{\mathbf{L}} \cdot \frac{d \mathbf{J}}{d t}\right)_{N} & =\frac{8 G^{2} m \bar{L}}{5 c^{5} \mu r^{5}}\left(2 \mu E r^{2}-3 \bar{L}^{2}\right), \\
\left(\hat{\mathbf{L}} \cdot \frac{d \mathbf{J}}{d t}\right)_{Q M} & =\frac{2 G^{2} m^{3}}{5 c^{5} \mu \bar{L}^{3} r^{7}} \sum_{i=1}^{2} p_{i}\left\{\sum_{n=1}^{3} b_{n} \cos \left(n \chi+\delta_{i}\right) \sin ^{2} \kappa_{i}+b_{4}\left(2-3 \sin ^{2} \kappa_{i}\right)\right\},
\end{aligned}
$$


with the coefficients $b_{k}$ given by

$$
\begin{aligned}
& b_{1}=3 \mu \bar{A} r\left(2 G m \mu^{3} E r^{3}-\mu E \bar{L}^{2} r^{2}-9 G m \mu^{2} \bar{L}^{2} r+8 \bar{L}^{4}\right), \\
& b_{2}=3\left(2 G^{2} m^{2} \mu^{5} E r^{4}-9 G^{2} m^{2} \mu^{4} \bar{L}^{2} r^{2}+22 \mu E \bar{L}^{4} r^{2}+10 G m \mu^{2} \bar{L}^{4} r-19 \bar{L}^{6}\right), \\
& b_{3}=\mu \bar{A} r\left(2 G m \mu^{3} E r^{3}+3 \mu E \bar{L}^{2} r^{2}-9 G m \mu^{2} \bar{L}^{2} r-24 \bar{L}^{4}\right) \\
& b_{4}=\bar{L} \bar{L}^{4}\left(-18 \mu E r^{2}-8 G m \mu^{2} r+15 \bar{L}^{2}\right) .
\end{aligned}
$$

A similar averaging procedure as in the case of the energy loss, gives here

$$
\begin{aligned}
\left\langle\frac{d L}{d t}\right\rangle & =\left\langle\frac{d L}{d t}\right\rangle_{N}+\left\langle\frac{d L}{d t}\right\rangle_{Q M}, \\
\left\langle\frac{d L}{d t}\right\rangle_{N} & =-\frac{4 G^{2} m(-2 \mu E)^{3 / 2}}{5 c^{5} \bar{L}^{4}}\left(14 E \bar{L}^{2}+15 G^{2} m^{2} \mu^{3}\right), \\
\left\langle\frac{d L}{d t}\right\rangle_{Q M} & =\frac{G^{2} m^{3} \mu(-2 \mu E)^{3 / 2}}{10 c^{5} \bar{L}^{8}} \sum_{i=1}^{2} p_{i}\left[D_{1} \sin ^{2} \kappa_{i} \cos \delta_{i}+D_{2}\left(2-3 \sin ^{2} \kappa_{i}\right)\right],
\end{aligned}
$$

where the coefficients $D_{k}$ are given below

$$
\begin{aligned}
& D_{1}=-6\left(62 E^{2} \bar{L}^{4}+211 G^{2} m^{2} \mu^{3} E \bar{L}^{2}+90 G^{4} m^{4} \mu^{6}\right), \\
& D_{2}=252 E^{2} \bar{L}^{4}+1200 G^{2} m^{2} \mu^{3} E \bar{L}^{2}+805 G^{4} m^{4} \mu^{6} .
\end{aligned}
$$

\section{Evolution of angles under radiation reaction}

The equations for the evolution under radiation reaction of the angles $\kappa_{i}=\cos ^{-1}\left(\hat{\mathbf{S}}_{\mathbf{i}} \cdot \hat{\mathbf{L}}\right),(i=1,2)$ and $\gamma=$ $\cos ^{-1}\left(\hat{\mathbf{S}}_{\mathbf{1}} \cdot \hat{\mathbf{S}}_{\mathbf{2}}\right)$ were derived in 19]:

$$
\begin{aligned}
\frac{d}{d t} \cos \gamma & \simeq 0 \\
\frac{d}{d t} \cos \kappa_{i} & \simeq\left(\frac{d}{d t} \cos \kappa_{i}\right)_{S O}+\left(\frac{d}{d t} \cos \kappa_{i}\right)_{S S}+\left(\frac{d}{d t} \cos \kappa_{i}\right)_{Q M} .
\end{aligned}
$$

The averaged $S O$ terms were computed previously and given in [17] and the averaged $S S$ terms in 19]. To the expression derived in [19] we have added the required quadrupole-monopole term:

$$
\left(\frac{d}{d t} \cos \kappa_{i}\right)_{Q M} \simeq \frac{1}{\bar{L}}\left(\hat{\mathbf{S}}_{\mathbf{i}}-\hat{\mathbf{L}} \cos \kappa_{i}\right) \cdot\left(\frac{d \mathbf{J}}{d t}\right)_{Q M},
$$

This is the new contribution to be computed in this subsection. For this purpose we rewrite the expression (3.27) as a function of the radial variables $r(\chi)$ and $\chi$ :

$$
\begin{aligned}
& \frac{1}{\bar{L}}\left(\hat{\mathbf{S}}_{\mathbf{i}}-\hat{\mathbf{L}} \cos \kappa_{i}\right) \cdot\left(\frac{d \mathbf{J}}{d t}\right)_{Q M}= \frac{3 G^{2} m^{3}}{10 c^{5} \mu \bar{L}^{2} r^{7}} \sin \kappa_{i} \sum_{j=1}^{2} p_{j} \sin 2 \kappa_{j}\left\{\sum_{n=1}^{3} u_{n} \cos \left(n \chi+\frac{\delta_{i}+\delta_{j}}{2}\right)\right. \\
&\left.\quad+u_{4} \sin \chi \sin \left(\frac{\delta_{i}-\delta_{j}}{2}\right)+u_{5} \cos \left(\frac{\delta_{i}-\delta_{j}}{2}\right)\right\}, \\
& \\
& u_{1}=-u_{3}=\mu \bar{A} r\left(2 \mu E r^{2}-3 \bar{L}^{2}\right), \\
& u_{2}=-2 \bar{L}^{2}\left(G m \mu^{2} r+3 \bar{L}^{2}\right), \\
& u_{4}=2 \mu \bar{A} r\left(2 \mu E r^{2}-5 \bar{L}^{2}\right), \\
& u_{5}=2 \bar{L}^{2}\left(4 \mu E r^{2}+G m \mu^{2} r-5 \bar{L}^{2}\right) .
\end{aligned}
$$

The residue theorem yields the averages: 


$$
\begin{gathered}
\left\langle\frac{d \gamma}{d t}\right\rangle=0 \\
\left\langle\frac{d \kappa_{i}}{d t}\right\rangle=\left\langle\frac{d \kappa_{i}}{d t}\right\rangle_{S O}+\left\langle\frac{d \kappa_{i}}{d t}\right\rangle_{S S-\text { self }}+\left\langle\frac{d \kappa_{i}}{d t}\right\rangle_{S S}+\left\langle\frac{d \kappa_{i}}{d t}\right\rangle_{Q M} \\
\left\langle\frac{d \kappa_{i}}{d t}\right\rangle_{S O} \quad \text { given by Eq. (4.4) of }[1] \\
\left\langle\frac{d \kappa_{i}}{d t}\right\rangle_{S S-\text { self }} \quad \text { given by Eq. (4.7) of [19] } \\
\left\langle\frac{d \kappa_{i}}{d t}\right\rangle_{S_{1} S_{2}} \quad \text { given by Eq. (4.8) of }[19], \\
\left\langle\frac{d \kappa_{i}}{d t}\right\rangle_{Q M}=\frac{3 G^{2} m^{3} \mu(-2 \mu E)^{3 / 2}}{10 c^{5} \bar{L}^{9}} \sum_{j=1}^{2} p_{j} \sin 2 \kappa_{j}\left[V_{1} \cos \left(\frac{\delta_{i}+\delta_{j}}{2}\right)+V_{2} \cos \left(\frac{\delta_{i}-\delta_{j}}{2}\right)\right],
\end{gathered}
$$

where the coefficients $V_{1-3}$ are:

$$
\begin{aligned}
& V_{1}=40 E^{2} \bar{L}^{4}+90 G^{2} m^{2} \mu^{3} E \bar{L}^{2}+35 G^{4} m^{4} \mu^{6} \\
& V_{2}=48 E^{2} \bar{L}^{4}+140 G^{2} m^{2} \mu^{3} E \bar{L}^{2}+70 G^{4} m^{4} \mu^{6} .
\end{aligned}
$$

For consistency in notation the replacements $L \rightarrow \bar{L}$ and $A_{0} \rightarrow \bar{A}$ should be carried out in Eq. (4.4) of [17] .

Equations (3.30)-(3.35) give the complete evolution under radiation reaction of the angles characterizing the relative orientation of the spin and orbital angular-momentum vectors up to second post-Newtonian order.

\section{CONCLUDING REMARKS}

As the main result we have derived the second post-Newtonian order quadrupole-monopole contribution to the radiation reaction for the energy, the magnitude of the orbital angular momentum, and the angular variables characterizing the relative orientation of angular momenta of a coalescing binary system. This was possible by introducing a suitable angular average $\bar{L}$ together with the generalized true and eccentric anomaly parametrizations for the quadrupole-monopole perturbation of the Keplerian motion.

The secular losses of $E$, Eqs. (3.8)-(3.10) and of $L$, Eqs. (3.21)-(3.23), complementing the corresponding spin-orbit terms of Ref. [17] (with the replacements $L \rightarrow \bar{L}$ and $A_{0} \rightarrow \bar{A}$ ), the spin-spin terms of Ref. [18], and the post-Newtonian terms of Ref. [11] give the total radiation reaction up to second post-Newtonian order.

All contributions to the secular evolutions of the angles $\kappa_{i}$ and $\gamma$ were listed in the Sec III.C. They are of either spin or quadrupolar origin.

Finally, we compare our result for the energy loss with the one given by Poisson in Ref. [20] for circular orbits. For this we need to take the circular orbit limit of Eqs. (3.8)-(3.11). By the circular orbit limit in Ref. [20] is meant a perturbation of a circular Keplerian orbit. This, however, cannot be immediately imposed in our formalism, relying on heavy use of conserved quantities of the perturbed motion. It is certainly impossible to impose the circularity conditions for the unperturbed orbit as $E_{N}=E-E_{Q M}=-G m \mu / 2 r_{0}$ and $L_{N}^{2}=L^{2}(\chi)=(\bar{L}+\delta L)^{2}=G m \mu^{2} r_{0}$ ( $r_{0}$ being the radius of the unperturbed orbit) due to the fact that $E_{Q M}$ and $\delta L$ are not constants, while $E, \bar{L}$ and $r_{0}$ are. This is related to the remark of [2] that the orbits cannot be circular in the strict sense. However, we can impose the circularity conditions in an average sense. As the angular average of $\delta L$ vanishes, we obtain:

$$
\begin{aligned}
& \bar{E}_{N}=E-\bar{E}_{Q M}=-\frac{G m \mu}{2 r_{0}}, \\
& \bar{L}_{N}^{2}=\bar{L}^{2}=G m \mu^{2} r_{0} .
\end{aligned}
$$

These values inserted in the angular average of $E_{Q M}$ (which is already of second PN order) give

$$
\bar{E}_{Q M}=\frac{G m^{3} \mu}{4 r_{0}^{3}} \sum_{i=1}^{2} p_{i}\left(3 \cos ^{2} \kappa_{i}-1\right) .
$$

Expressing $E$ and $\bar{L}$ from Eqs. (4.1) and (4.2) and inserting them into the expressions (3.8)-(3.11) for the energy loss we find the radiative energy loss for the above defined circular orbits: 


$$
\left\langle\frac{d E}{d t}\right\rangle=-\frac{32 G^{4} m^{3} \mu^{2}}{5 c^{5} r_{0}^{5}}\left[1-6 \frac{m^{2}}{r_{0}^{2}} \sum_{i=1}^{2} p_{i}\left(3 \cos ^{2} \kappa_{i}-1\right)\right] .
$$

Employing the relation between the radius of the unperturbed circular orbit $r_{0}$ and the average velocity of the perturbed orbit $\langle v\rangle$, deducible from the expressions given in Ref. [20]:

$$
r_{0}=\frac{G m}{\langle v\rangle^{2}}\left[1-\frac{\langle v\rangle^{4}}{G^{2}} \sum_{i=1}^{2} p_{i}\left(3 \cos ^{2} \kappa_{i}-1\right)\right],
$$

we find that the averaged energy loss for circular orbits agrees with Eq. (22) of Ref. [20].

\section{ACKNOWLEDGMENTS}

This work was supported by the Hungarian Higher Education and Research Foundation (AMFK). The algebraic package REDUCE was employed in some of the computations.

[1] A. Abramovici et al., Science 256, 325 (1992).

[2] C. Bradaschia et al., Nucl. Instrum. Methods Phys. Res. A 289, 518 (1990).

[3] J. Hough, in Proceedings of the Sixth Marcell Grossmann Meeting, edited by H. Sato and T. Nakamura (World Scientific, Singapore, 1992), p. 192.

[4] K. Kuroda et al., in Proceedings of International Conference on Gravitational Waves: Sources and Detectors, edited by I. Ciufolini and F. Fidecaro (World Scientific, Singapore, 1997), p. 100.

[5] P. Bender, I. Ciufolini, K. Danzmann, W. M. Folkner, J. Hough, D. Robertson, A. Rüdiger, M. C. W. Sandford, R. Schilling, B. F. Schutz, R. Stebbins, T. Sumner, P. Touboul, S. Vitale, H. Ward, and W. Winkler: "LISA: Pre-Phase A Report (MPQ 208)" (Max-Planck Institut für Quantenoptik, Garching, Germany, 1996).

[6] B. F. Schutz, "Proceedings of the 1997 Alpbach Summer School on Fundamental Physics in Space", edited by A. Wilson, gr-qc/9710079.

[7] P. C. Peters, Phys. Rev. 136, B1224 (1964).

[8] G. D. Quinlan and S. L. Shapiro, Astrophys. J. 321, 199 (1987)

[9] D. Hills and P. L. Bender, Astrophys. J. Lett. 445, L7 (1995).

[10] K. Martel and E. Poisson, Phys. Rev. D 60, 124008 (1999).

K. Martel, Detection of Gravitational Waves from Eccentric Compact Binaries, gr-qc/9908043.

[11] A. Gopakumar and B. R. Iyer, Phys. Rev. D 56, 7708 (1997).

[12] B. M. Barker and R. F. O'Connell, Phys. Rev. D 12, 329 (1975). Comparison is immediate by changing their notations $M, \mathbf{n}^{(\mathbf{i})}, J_{2}^{(i)}, I^{(i)}, \Delta I^{(i)}, \omega^{(i)}$ to $m, \hat{\mathbf{S}}_{\mathbf{i}},-m^{2} p_{i}, \Theta_{i}^{\prime},-Q_{i}, \Omega_{i}$ respectively.

[13] T. A. Apostolatos, C. Cutler, G. J. Sussman and K. S. Thorne, Phys. Rev. D 49, 6274 (1994).

[14] L. Kidder, C. Will, and A. Wiseman, Phys. Rev. D 47, 4183 (1993).

[15] L. Kidder, Phys. Rev. D 52, 821 (1995).

[16] R. Rieth and G. Schäfer, Class. Quantum Grav. 14, 2357 (1997).

[17] L. Á. Gergely, Z. Perjés, and M. Vasúth, Phys. Rev. D 58, 124001 (1998).

[18] L. Á. Gergely, Phys. Rev. D 61, 024035 (2000).

[19] L. Á. Gergely, Phys. Rev. D 62, 024007 (2000).

[20] E. Poisson, Phys. Rev. D 57, 5287 (1998).

[21] L. Á. Gergely, Z. Perjés, and M. Vasúth, Astrophys. J. Suppl. Ser. 126, 79 (2000).

[22] H. Goldstein, Classical Mechanics, 2nd ed. (Addison-Wesley, Reading, MA, 1980), Chap. 5.

[23] H. Tagoshi, A. Ohashi, and B. J. Owen, Phys. Rev. D 63, 044006 (2001).

[24] L. Á. Gergely, Z. Perjés, and M. Vasúth, Phys. Rev. D 57, 3423 (1998). 